\title{
BMJ Open Maximising comfort: how do patients describe the care that matters? A two- stage qualitative descriptive study to develop a quality improvement framework for comfort-related care in inpatient settings
}

Cynthia Wensley (D , ${ }^{1}$ Mari Botti, ${ }^{2}$ Ann McKillop, ${ }^{1}$ Alan F Merry ${ }^{3,4}$

To cite: Wensley C, Botti M, McKillop A, et al. Maximising comfort: how do patients describe the care that matters? A two-stage qualitative descriptive study to develop a quality improvement framework for comfort-related care in inpatient settings. BMJ Open 2020;10:e033336. doi:10.1136/ bmjopen-2019-033336

- Prepublication history and additional material for this paper are available online. To view these files, please visit the journal online (http://dx.doi. org/10.1136/bmjopen-2019033336).

Received 31 July 2019 Revised 17 February 2020 Accepted 24 February 2020

D) Check for updates

(C) Author(s) (or their employer(s)) 2020. Re-use permitted under CC BY-NC. No commercial re-use. See rights and permissions. Published by BMJ.

For numbered affiliations see end of article.

Correspondence to Dr Cynthia Wensley; c.wensley@auckland.ac.nz

\section{ABSTRACT}

Objective To develop a multidimensional framework representing patients' perspectives on comfort to guide practice and quality initiatives aimed at improving patients' experiences of care.

Design Two-stage qualitative descriptive study design. Findings from a previously published synthesis of 62 studies (stage 1) informed data collection and analysis of 25 semistructured interviews (stage 2) exploring patients' perspectives of comfort in an acute care setting. Setting Cardiac surgical unit in New Zealand. Participants Culturally diverse patients in hospital undergoing heart surgery.

Main outcomes A definition of comfort. The Comfort ALways Matters (CALM) framework describing factors influencing comfort.

Results Comfort is transient and multidimensional and, as defined by patients, incorporates more than the absence of pain. Factors influencing comfort were synthesised into 10 themes within four inter-related layers: patients' personal (often private) strategies; the unique role of family; staff actions and behaviours; and factors within the clinical environment.

Conclusions These findings provide new insights into what comfort means to patients, the care required to promote their comfort and the reasons for which doing so is important. We have developed a definition of comfort and the CALM framework, which can be used by healthcare leaders and clinicians to guide practice and quality initiatives aimed at maximising comfort and minimising distress. These findings appear applicable to a range of inpatient populations. A focus on comfort by individuals is crucial, but leadership will be essential for driving the changes needed to reduce unwarranted variability in care that affects comfort.

\section{INTRODUCTION}

Championing patients' need for comfort was central to the origins of person-centred care organisations such as the Picker Institute ${ }^{1}$ and Planetree. ${ }^{2}$ Within the executive summary of
Strengths and limitations of this study

- A comprehensive conceptual framework developed from an integrative review of 62 studies (14 theoretical and 48 qualitative) focused the exploration of patients' perspectives on comfort in an acute care setting.

- The definition of comfort (the state) and description of influencing factors (processes of care) were developed using qualitative methods aimed at understanding how comfort and comforting is perceived and experienced by patients.

- The study reported on here is the first that has set out to explore a cultural dimension of comfort via purposive sampling of culturally diverse patients.

- Peer debriefing, Māori and Pacific consultation, prolonged engagement, negative case analysis and triangulation promote credibility.

- The two-stage approach enabled development (stage 1) and then refinement (stage 2) of themes and operational definitions that capture the broad influences on comfort in one unifying framework. However, identifying context-specific detail is required for application.

the Institute of Medicine's landmark report 'To Err is Human' is stated, 'it is not acceptable for patients to be harmed by the health care system that is supposed to offer healing and comfort' ${ }^{3}$ (p. 3). Hippocrates' quote 'To cure sometimes, to relieve often, to comfort always' is familiar to many. More recently, the 2012 National Institute for Health and Care Excellence Patient Experience Guideline identified 'comfort' as one of seven outcomes of a good patient experience. ${ }^{4}$ Informed by the work of Gerteis and colleagues, ${ }^{5}$ promoting physical comfort became a core aspect of person-centred care frameworks. ${ }^{47}$ Comfort is also regarded as holistic 
and multidimensional, ${ }^{8-12}$ associated with concepts that are hallmarks of a caring and humane society such as dignity, empathy, kindness and compassion. ${ }^{13-15}$ This notion of comfort fits with evidence provided by patients and family during the Mid-Staffordshire Inquiry ${ }^{16}$ where good-and bad-care was described in terms of comfort, discomfort, comforting or feeling/looking uncomfortable. As such, comfort, or lack of it, is a defining aspect of patients' experiences and an indication of the overall quality and safety of care. A service that fails to provide high-quality care that includes the promotion of comfort, or recognise avoidable suffering as a source of harm, means that patients and their family have been let down by those who are meant to care for them. ${ }^{3}{ }^{36-22}$ Overall, reducing unwarranted variability in care important for comfort is a crucial aspect of quality person-centred care in contemporary healthcare settings.

But what is comfort, and what care matters to patients? Differing definitions ${ }^{8101123}$ and perspectives on comfort depicted in person-centred frameworks ${ }^{67}$ and concept analyses $^{8-12}$ highlight that this concept is poorly defined for practice and quality improvement. In particular, the absence of a framework incorporating all that is relevant from patients' perspectives ${ }^{24}$ risks provider-centric improvement that fails to deliver the care that matters. The purpose of this research was to develop a multidimensional framework representing patients' perspectives on comfort that can be applied in a range of healthcare settings to guide practice and quality improvement initiatives aimed at improving patients' experiences of care.

\section{METHOD}

A two-stage qualitative descriptive study design ${ }^{25}$ was used to explore patients' perspectives on comfort and its influencing factors. This design is known for producing 'findings closer to the data ${ }^{25}$ (p. 78) and was considered appropriate for generating findings that could be translated into practice. In stage 1, data from 62 studies exploring the concept of comfort in healthcare settings were synthesised into a conceptual framework representing patients' perspectives on comfort. ${ }^{24}$ Integrative review methods facilitated identification of multiple dimensions of comfort that appeared relevant. This framework informed the study reported here, which explored the concept of comfort in patients undergoing heart surgery. Heart surgery can be physically and emotionally distressing, ${ }^{26}$ therefore exploring patients' perspectives on comfort and comforting care in a cardiac surgical setting was ideal. In summary, our two-stage approach enabled development (stage 1) and then refinement (stage 2) of a framework representing patients' perspectives on factors influencing comfort.

\section{Patient and public involvement}

We used an exploratory method of data collection to better understand patients' perspectives and experiences of care. Questions were informed by a conceptual framework developed from studies also exploring patients' perspectives. Patients were not directly involved in the design of this research. However, cultural advisors provided advice that facilitated Māori and Pacific recruitment, led to refinements of the interview procedure and supported accurate representation of Māori and Pacific worldviews. The acceptability of the interview process and questions were tested in five pilot interviews involving patients of Māori, Pacific and New Zealand European (NZE) ethnicities. As part of the informed consent process, participating patients were offered the opportunity to review their interview transcript and feedback on its accuracy via a prepaid postage return of the hard copy or follow-up phone call. Presentations of the findings have been made in order for our results to benefit future patients and to guide research aimed at improving patient experience.

\section{Site and setting}

The study was conducted in a 47-bed cardiac surgical unit in a publicly funded hospital in Auckland, New Zealand.

\section{Participant selection}

Purposive sampling was used to access and invite participation from culturally diverse patients. Sampling aimed for one-third each of Māori (the tangata whenua or indigenous people of Aotearoa/New Zealand), NZE and Pacific people (people who migrated from, or who identify with, the Pacific Islands) to enable exploration of a cultural dimension of comfort. Inclusion criteria were: postoperative day 4 or 5 after operations classified as coronary artery bypass graft or valve replacement/repair; age 16 years or older; English speaking; transferred from the intensive care unit postoperative day 1; an expectation of discharge at or before eight postoperative days; sedation score of 0 (awake and alert) or 1 (mild sedation and easy to rouse); and ability to provide consent. Participants were identified in consultation with a senior nurse and then invited to participate by one researcher (CW) who emphasised her non-employee status. Informed consent was obtained. One experienced researcher $(\mathrm{CW})$ conducted all interviews. Data saturation was sought regarding understanding how perspectives on comfort differed by ethnicity.

\section{Data collection}

Semistructured patient interviews explored: (1) what comfort meant to patients from which a definition of comfort was to be developed and (2) factors within the care setting that influenced comfort, that is, what care mattered to patients. Questions exploring influencing factors were informed by the conceptual framework ${ }^{24}$ (see online supplementary file 1, patient interview guide). Patients were not asked directly if the broad influences identified a priori were important for comfort. Pilot testing indicated this approach risked bias towards affirmative responses and less nuanced data. Rather, patients were asked about aspects of care related to conceptual framework themes, and responses were probed to determine the influences 
on comfort. Negative case analysis (searching for disconfirming evidence) was used throughout data collection and analysis. ${ }^{27}$ The final interview question gave participants the opportunity to describe influences on comfort that may have been missed. Interview settings were patients' single rooms $(n=7)$, a quiet room on the ward $(n=13)$ or patients' four-bedded room $(n=5)$ the latter being participants' preference. Interview durations were between 23 and $66 \mathrm{~min}$ (average $43 \mathrm{~min}$ ) and similar between ethnicities (see online supplementary file 2, characteristics of patients). Audiorecorded interviews were transcribed verbatim. Participants were sent a copy of their interview transcript and given the opportunity to comment on accuracy and content.

\section{Data analysis}

Analysis was sequential. General inductive $\operatorname{method}^{28}$ was used to analyse data contributing to a definition of comfort. Inductive analysis gave some assurance that the definition of comfort was data derived and developed without undue researcher influence. ${ }^{28} 29$ Analysis involved: close reading of the transcribed text; creation of specific and then general (higher level) categories from patients' description of comfort or derivatives of the word comfort (comforting, comfortable, uncomfortable and discomfort); and revision and refinement until four overall categories capturing the essence of what comfort feels like to patients were identified. Categories were summarised into a definition of comfort.

Thematic analysis ${ }^{29}$ and Framework method ${ }^{30}$ were used to analyse data related to influences on comfort using both deductive and inductive analysis. Deductive analysis tested the relevance of the conceptual framework to patients' perspectives. Inductive analysis was important for enabling us to identify any new themes ${ }^{29}{ }^{30}$ within patient interview data. The steps involved were:

- Familiarisation with the transcribed texts. The definition of comfort was used to identify and begin coding patient interview data relevant to influences on comfort. Familiarisation involved careful consideration of the overall 'fit' of that data to the conceptual framework themes. ${ }^{24}$

- Constructing an initial thematic framework from the conceptual framework headings ${ }^{24}$ building in themes and subthemes identified within the coded data. Some codes were derived from theme definitions developed a priori, ${ }^{24}$ while other codes were developed inductively from the data.

- Indexing and sorting, in which data were systematically sorted into the thematic framework.

- Reviewing data extracts, checking for coherence between codes and refining the thematic framework accordingly.

- Data summary and display; matrices of distilled coded text were developed for each subtheme to enable data to be easily compared between participants and between ethnic groups.

- Abstraction and interpretation of the data; multiple and inter-related factors influencing patient comfort were identified. A careful comparison between stage $1^{24}$ and stage 2 findings was made to determine transferability beyond the cardiac surgical setting. ${ }^{31}$

Data were managed using NVivo Version 10 software. One researcher (CW) coded all data. Coding decisions were discussed at regularly scheduled meetings (MB, AM, CW). Peer debriefing ${ }^{27}$ occurred throughout all stages of data analysis. Discussion and refinement of themes and subthemes occurred until consensus was reached. Consultation with Māori and Pacific healthcare staff ensured that the recruitment process, interview procedure and data analysis promoted participation of Māori and Pacific patients and accurate representation of their worldview. We used the SRQR checklist when writing our report. $^{32}$

\section{RESULTS}

Twenty-five participants were interviewed on either day 4 $(72 \%)$ or day $5(28 \%)$ after surgery. Eight patients selfidentified as Māori, 7 as Pacific people and 10 as NZE. Median age was 63 years (range $30-85$ ) and $64 \%$ were men. Fourteen patients underwent coronary artery bypass graft, 10 underwent valve replacement/repair $(n=10)$ and 1 patient underwent both (online supplementary file 2). Fifteen patients declined participation for reasons outlined in online supplementary file 3.

\section{Comfort: a universal concept}

Perspectives on comfort reported by patients in primary studies ${ }^{24}$ were similar to those held by patients undergoing heart surgery. As such, comfort is regarded as having universal relevance, and the findings presented here appear applicable to a range of inpatient populations.

\section{Patients' perspectives on comfort}

Patients' perspectives on comfort are summarised in the following definition:

Comfort is a transient and dynamic state characterised by ease from pain, emotional and physical distress and an emerging sense of positivity, safety, strength and acceptance of one's situation that is underpinned and sustained by feeling valued, cared for, confident and accepting treatment by choice. Total comfort is elusive; rather, patients seek to be as comfortable as they can be under the circumstances of their healthcare interaction.

Underpinning our definition are the following four senses of comfort that were identified in the patient interview data:

- 'Relief (ease) from pain, emotional and physical distress'.

- 'Feeling positive, safe and stronger'.

- 'Feeling confident, in control, accepting treatment and care by choice'.

- 'Feeling cared for, valued; connecting positively to people and place'. 


\section{$\longrightarrow$ Comfort}

Comfort is a transient and dynamic state characterised by ease from pain, emotional and physical distress and an emerging sense of positivity, safety, strength and acceptance of one's situation that is underpinned and sustained by feeling valued, cared for, confident and accepting treatment by choice.

Total comfort is elusive; patients seek to be as comfortable as they can be under the circumstances of their healthcare interaction.

\section{Influences on comfort}

$\checkmark \quad$ Self-comforting strategies; positive thinking, seeking (and finding) signs of safety and normality, self-care routines, developing acceptance (getting informed, planning, learning to trust).

$\checkmark \quad$ Culturally connected; finding cultural familiarity, sensing that one's cultural norms and values are understood and respected.

$\checkmark \quad$ Spiritually connected; sustaining personally significant spiritual or religious practices.

$\checkmark \quad$ Family; family have a unique ability to comfort arising from a shared culture and understanding.

$\checkmark \quad$ Symptom Management; all symptoms (not just pain).

Holistic Care and Assistance; non-pharmacological interventions to ease discomfort, help provided willingly.

$\checkmark \quad$ Engaged and Committed staff; staff presence, therapeutic comforting strategies tailored to individual need.

$\checkmark \quad$ Information and Participation; accurate information that is sensitively provided so that patients can prepare for what lies ahead. Opportunities to participate in, and personalise, care.

$\checkmark \quad$ Competent staff; perceived and actual

$\checkmark \quad$ Physical Facilities and Ambience; clean, well-equipped and family-friendly. Patients can control their personal space (lights, noise). Staff project an ambience of positivity and caring, are seen to be working as a cohesive team and have time for ALL patients' needs.

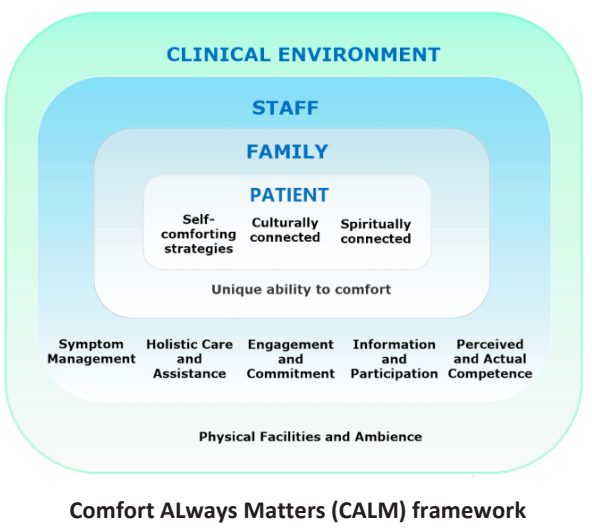

Figure 1 Patients' perspectives on comfort and influencing factors; the Comfort ALways Matters (CALM) framework

Figure 1 Patients' perspectives on comfort and influencing factors; the comfort always matters (CALM) framework.

\section{When is comforting care important?}

Patients' need for comforting care varied between individuals and could occur at any stage of the healthcare interaction. Common triggers were: the uncertainty and fear of treatment and planned procedures; pain, emotional and physical distress; feeling vulnerable, dependent and weak from functional loss and the accumulative effect of multiple symptoms; being in an unfamiliar environment; and missing home and family.

\section{Factors influencing patients' comfort}

Factors influencing comfort were complex but underpinned by 10 themes, as depicted in the conceptual framework that we had named the Comfort ALways Matters (CALM) framework ${ }^{24}$ (see figure 1 ). Themes occurred within four integrated layers: patients' personal strategies; the role of family; staff actions and behaviours; and factors within the clinical environment. The broad themes identified in stage 1 were consistent with those identified by patients undergoing heart surgery. Most theme names were retained. However, patient interview data led to a deeper, more nuanced understanding of these themes. Accordingly, the theme definitions presented here have been refined to better reflect: (1) the care that matters to patients, (2) the integrated nature of that care and (3) aspects of culturally responsive care that had not been previously identified. The theme related to family influences was renamed to reflect important ethnocultural differences in the way family comfort. The essence of each theme and their unified influence on patients' sense of comfort is portrayed in figure 1 . Operational definitions, subthemes and illustrative quotes for all themes are provided in tables 1-4. Themes within each layer are now discussed.

\section{Patients' personal strategies}

The first (inner) layer of the CALM framework relates to patients' use of personal (often private) strategies to promote comfort and ease distress (see table 1). Three themes were identified within this layer, the first describes patients use of 'Self-comforting strategies' during times of distress and uncertainty. Strategies were categorised under four subthemes, which were maintaining positivity; looking for reassuring signs of safety through surveillance of self and others; easing distress using distraction or self-care routines; and developing acceptance of one's situation by, for example, getting informed, planning and learning to 


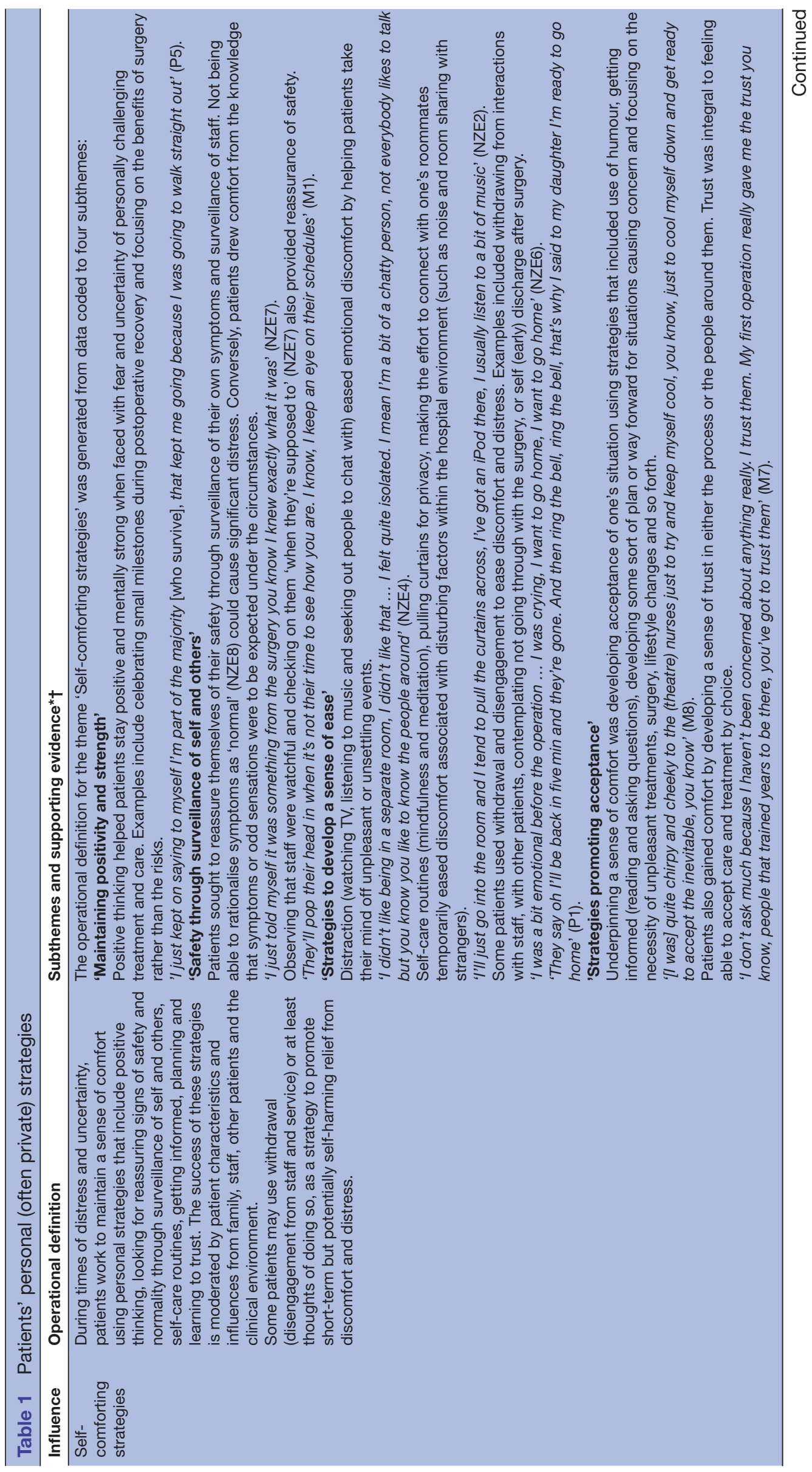




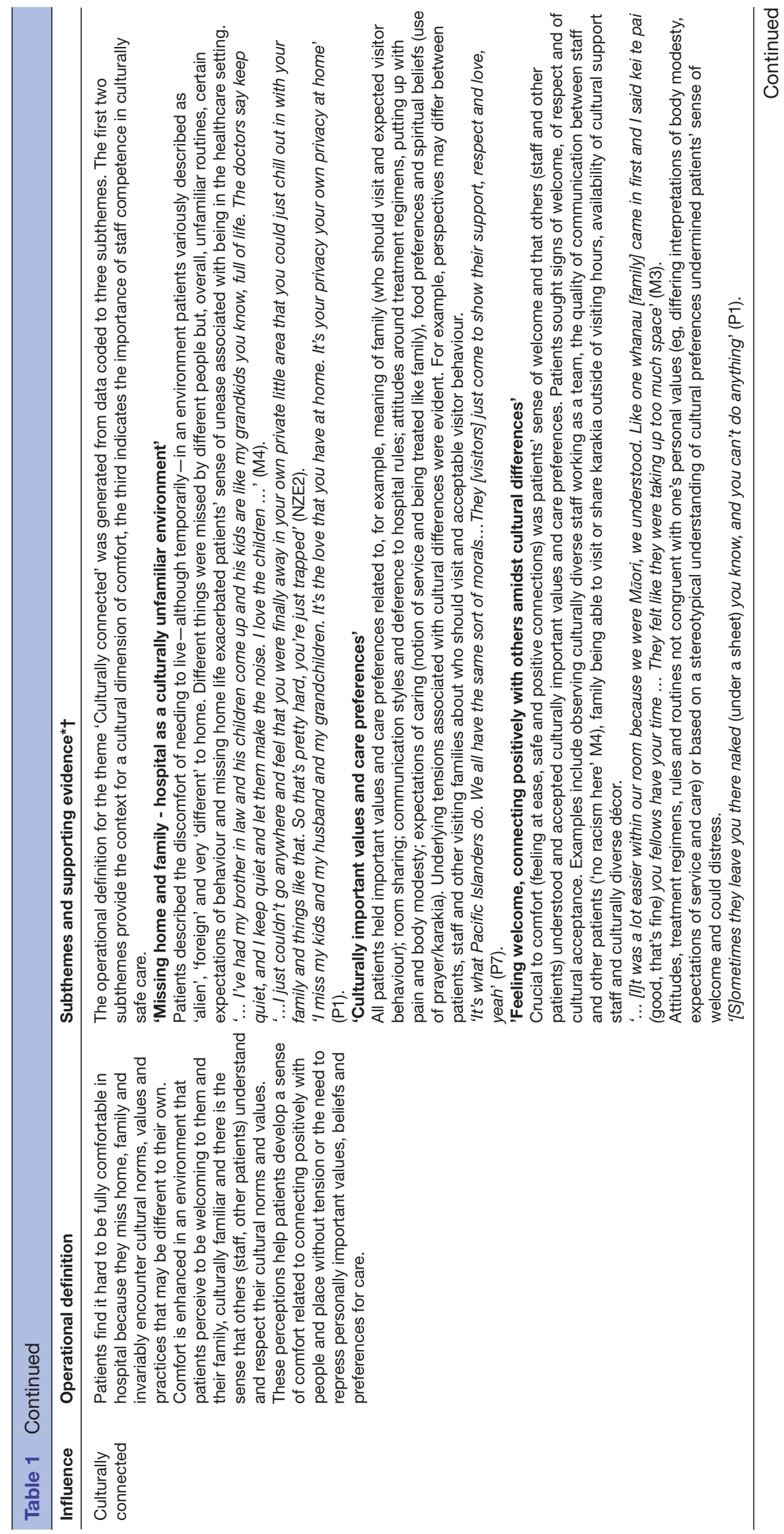


trust (see table 1, self-comforting strategies). The second theme was about comfort arising from feeling 'Culturally connected', which related to seeking cultural familiarity, and feeling that one's cultural norms and values were understood and respected by others (see table 1, culturally connected). The third theme described comfort gained from feeling 'Spiritually connected'. For some patients, connecting to a higher power through personally significant spiritual or religious practices was comforting (see table 1 , spiritually connected). In all three themes, actions and behaviour of family, staff,and factors within the clinical environment moderated the success of these strategies.

Influence of Family

The second layer of the CALM framework related to the theme 'Family's unique ability to comfort' (see table 2, family's unique ability to comfort). Exploring family comforting in a culturally diverse sample identified that family's unique connection with patients was pivotal to their ability to comfort. Differences in the way families comforted (whether by shared prayer/karakia, bringing food in and encouraging trust) and who comforted (immediate or extended family) were identified between ethnicities. A shared culture and understanding appeared to underpin the differences in family-initiated comforting observed.

Overall, family were an important buffer to the unfamiliar clinical environment. Additionally, for most patients, having loved ones near and connecting with those who know them best and whom they trust promoted positivity and acceptance of care. Family-initiated comforting activities also included providing holistic care and practical support. However, family could also distress. Patients expressed concern for the safety and well-being of family members and worried about being a burden. Conflict between staff and family could undermine patients' confidence in treatment decisions. Positive family-staff relationships and family-friendly facilities are the most comforting scenario for patients. These examples demonstrate the integration between family - staffclinical environment layers that was better understood through stage 2 patient enquiry.

\section{Staff Actions and Behaviour}

The third layer of the CALM framework relates to the way staff actions and behaviour influence comfort. Five distinct but integrated themes were identified (see table 3). The first staff-related theme was effective 'Symptom Management', which was essential for all symptoms including but not limited to pain. Distressing symptoms varied considerably even in a relatively homogeneous group of patients; therefore, individualised management was important (see table 3 , symptom management).

The second staff-related theme, 'Holistic Care and Assistance' acknowledges the significant physical and emotional discomfort that can arise from the accumulative effect of symptoms, treatment side effects, unpleasant procedures and loss of functional ability. Holistic care involving multiple, non-pharmacological interventions was essential and complemented pharmacological symptom management. Help with personal care and basic tasks was crucial 


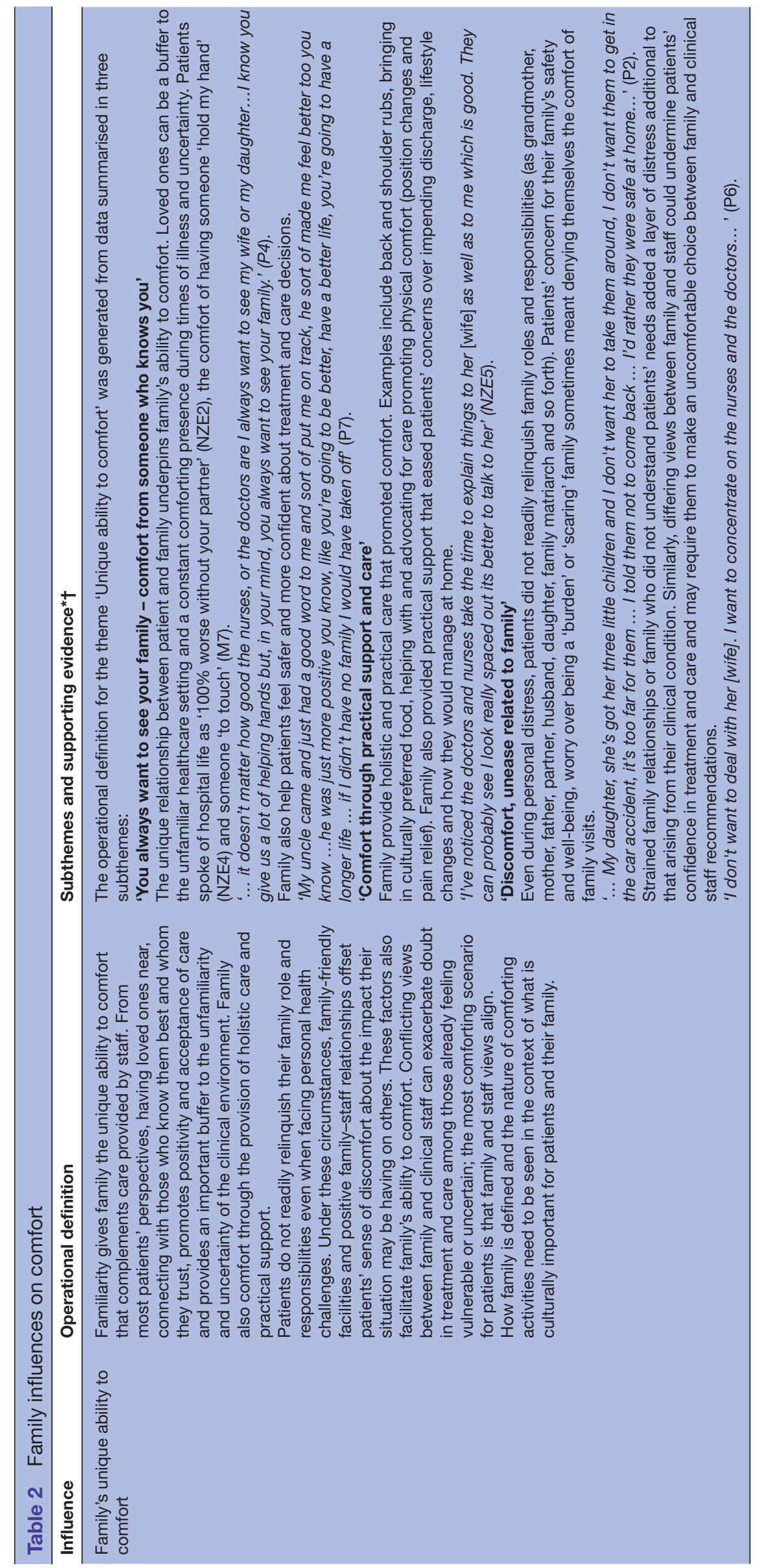

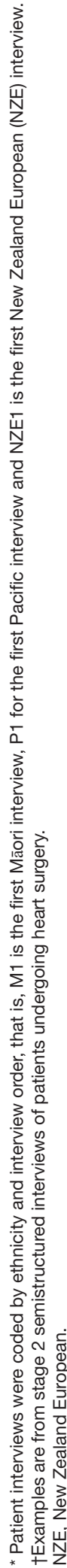




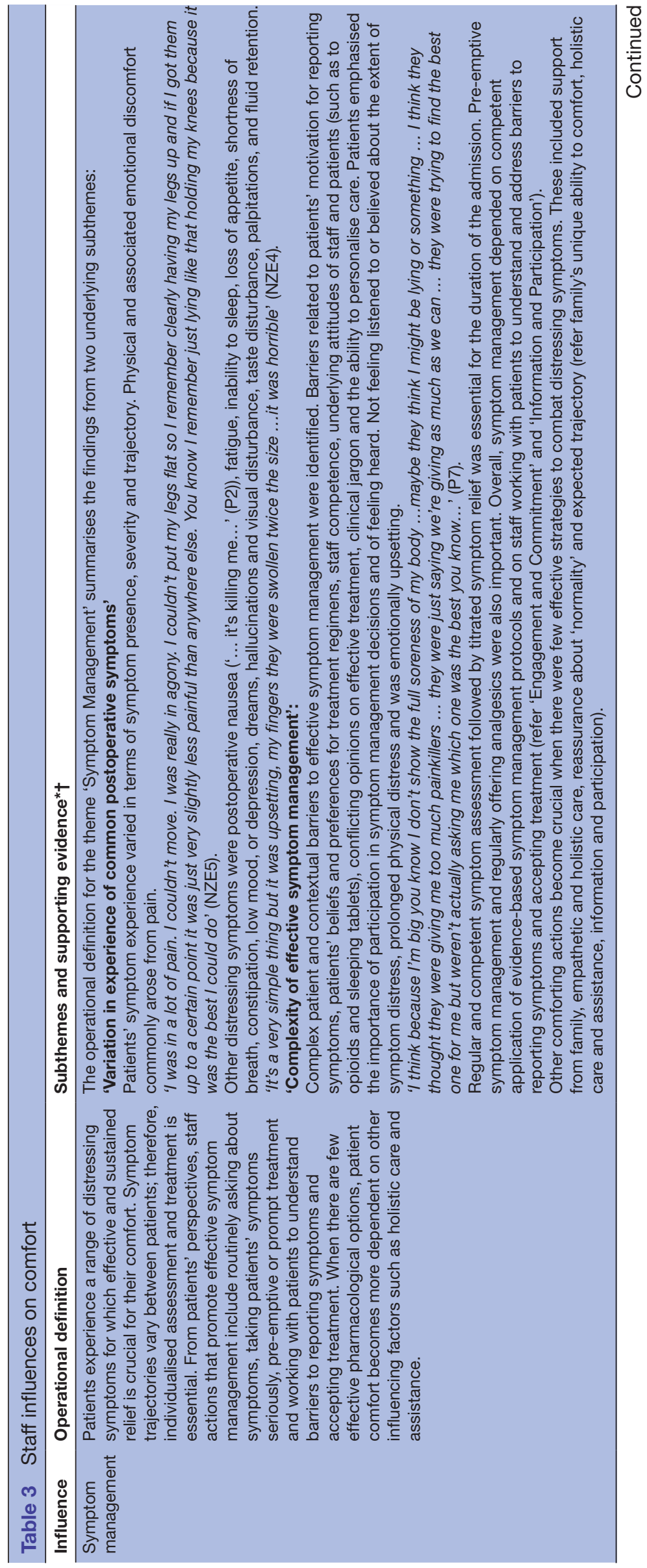




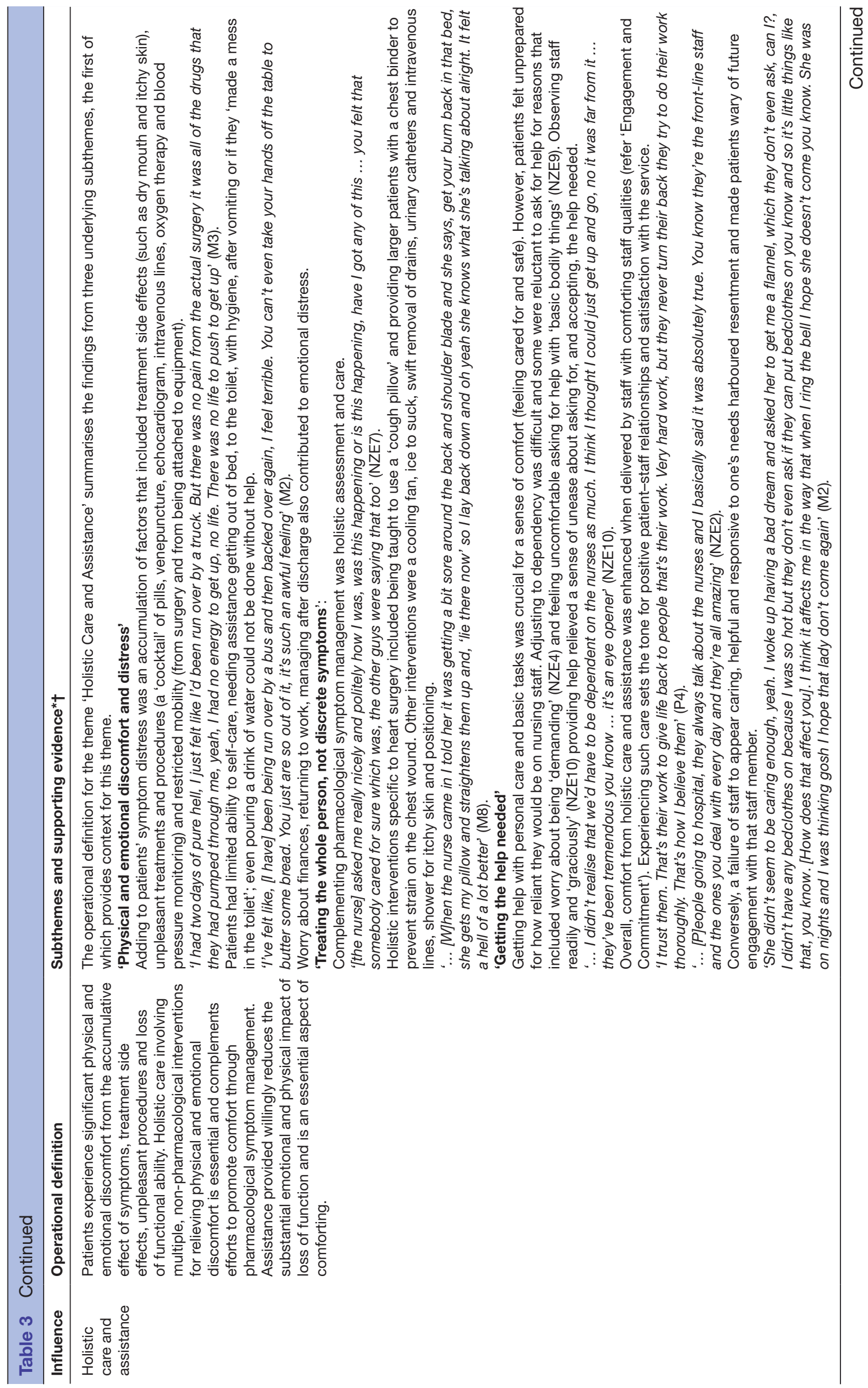




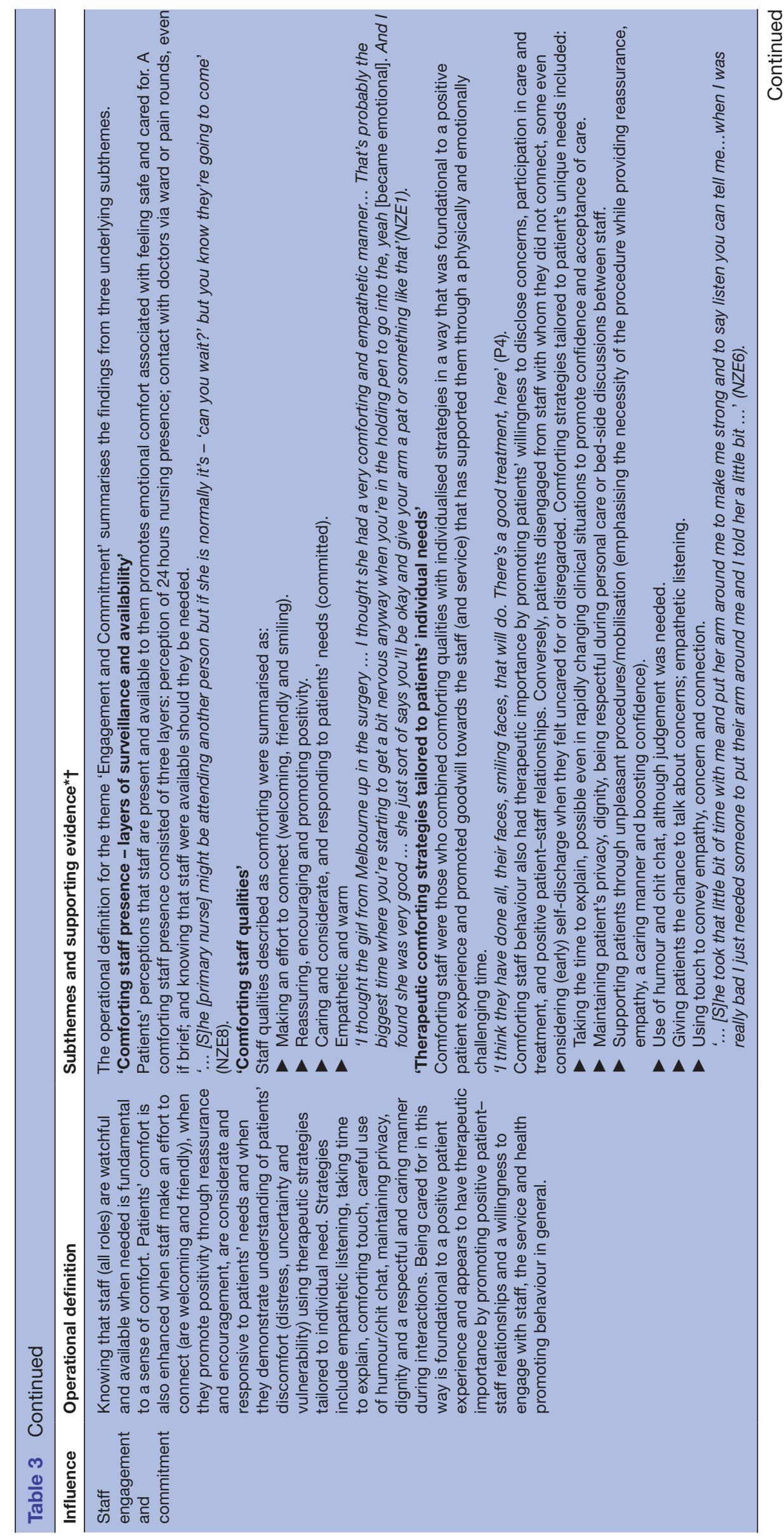




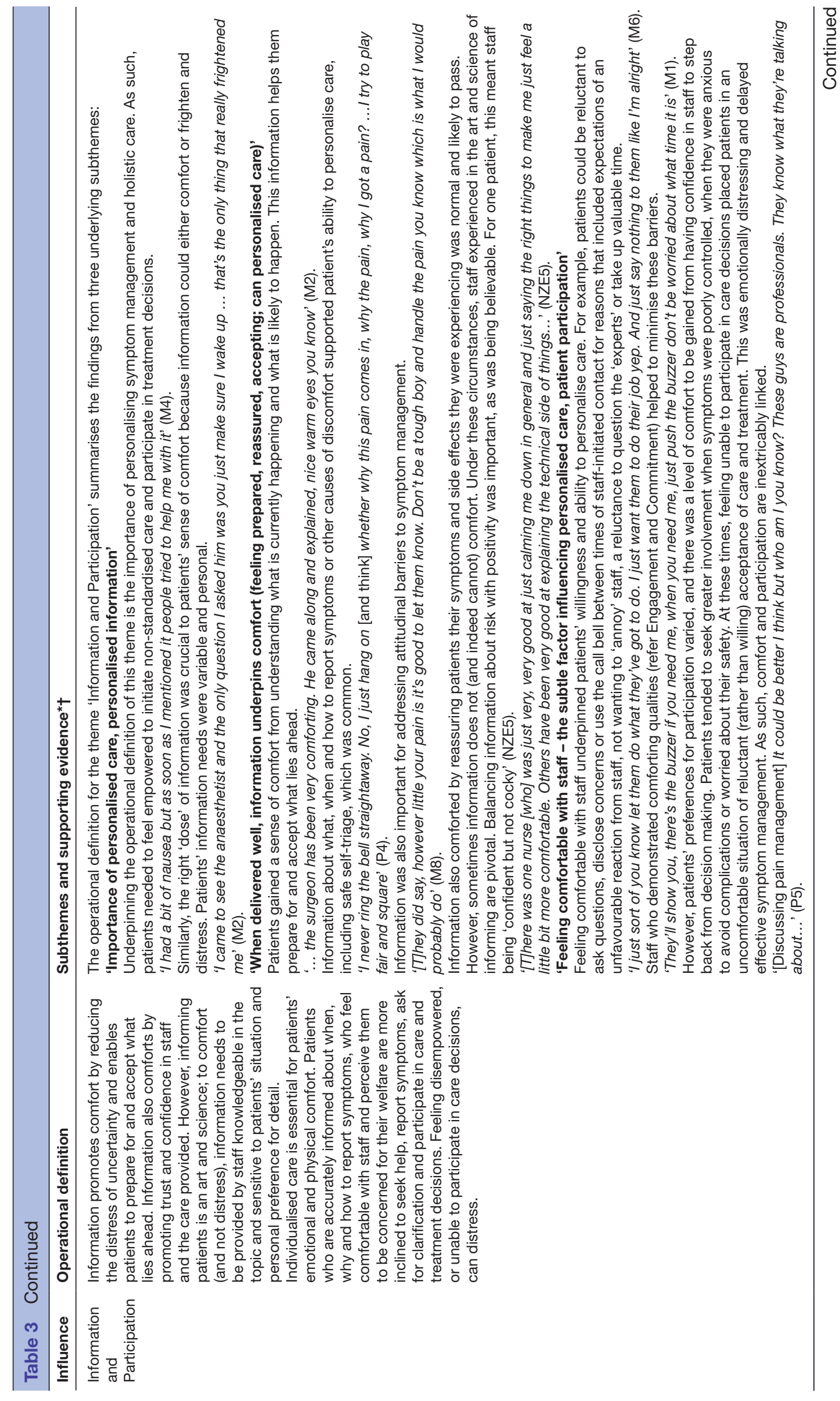


for a sense of comfort related to feeling cared for and safe (see table 3, holistic care and assistance).

Comfort from holistic care and assistance was enhanced when delivered by staff with qualities described in the third theme, 'Engagement and Commitment'. This theme relates to a sense of comfort arising from patients' perceptions that staff were engaged in, and committed to, their welfare. Staff presence was important, which encompassed: the perception of 24 hours nursing presence; contact with doctors via ward rounds; and knowing that staff were available should they be needed. Comforting staff qualities included making an effort to connect, providing reassurance, encouragement and responding to patients' discomfort or distress using therapeutic strategies tailored to individual need (see table 3 , engagement and commitment).

The fourth theme related to staff influence was 'Information and Participation', which influenced comfort in complex ways. When delivered well, information influenced comfort by enabling patients to feel prepared, reassured or, at least, accept the need for treatment and care. In addition, information and participation opportunities moderated patients' ability to personalise many aspects of care important for their comfort. For example, patients were more likely to seek help, disclose concerns or report symptoms when clearly informed about when, why and how to do so. Personalising care in this way also seemed more likely when patients felt comfortable with staff (refer to 'Engagement and Commitment'). Preferences for participation varied but feeling overlooked, or unable to participate in care decisions could distress (see table 3 , information and participation).

The fifth staff-related theme was 'Perceived and Actual Competence'. Perception of staff competence was comforting in the sense that patients felt at ease and confident in the care provided. Actual competence in all influences was crucial. Interview data indicated that all staff can influence comfort by being competent in their role while mindful that patients' need for comfort is individual and may occur at any stage of their healthcare experience (see table 3 , perceived and actual competence).

Factors within the clinical environment

The outer layer of the CALM framework relates to the theme 'Physical Facilities and Ambience', which summarises factors within the clinical environment that influence comfort (see table 4, physical facilities and ambience). Among the factors important here were an ambience of caring and positivity, observing that staff had time for all patients' needs, having control over one's personal space (lights and noise) and facilities that were clean, well-equipped and family-friendly.

\section{DISCUSSION}

Through a two-stage process commencing with an integrative review involving 62 studies $^{24}$ followed by semistructured patient interviews, we have: (1) defined patients' perspectives on comfort and (2) developed a multidimensional framework representing patients' perspectives on 


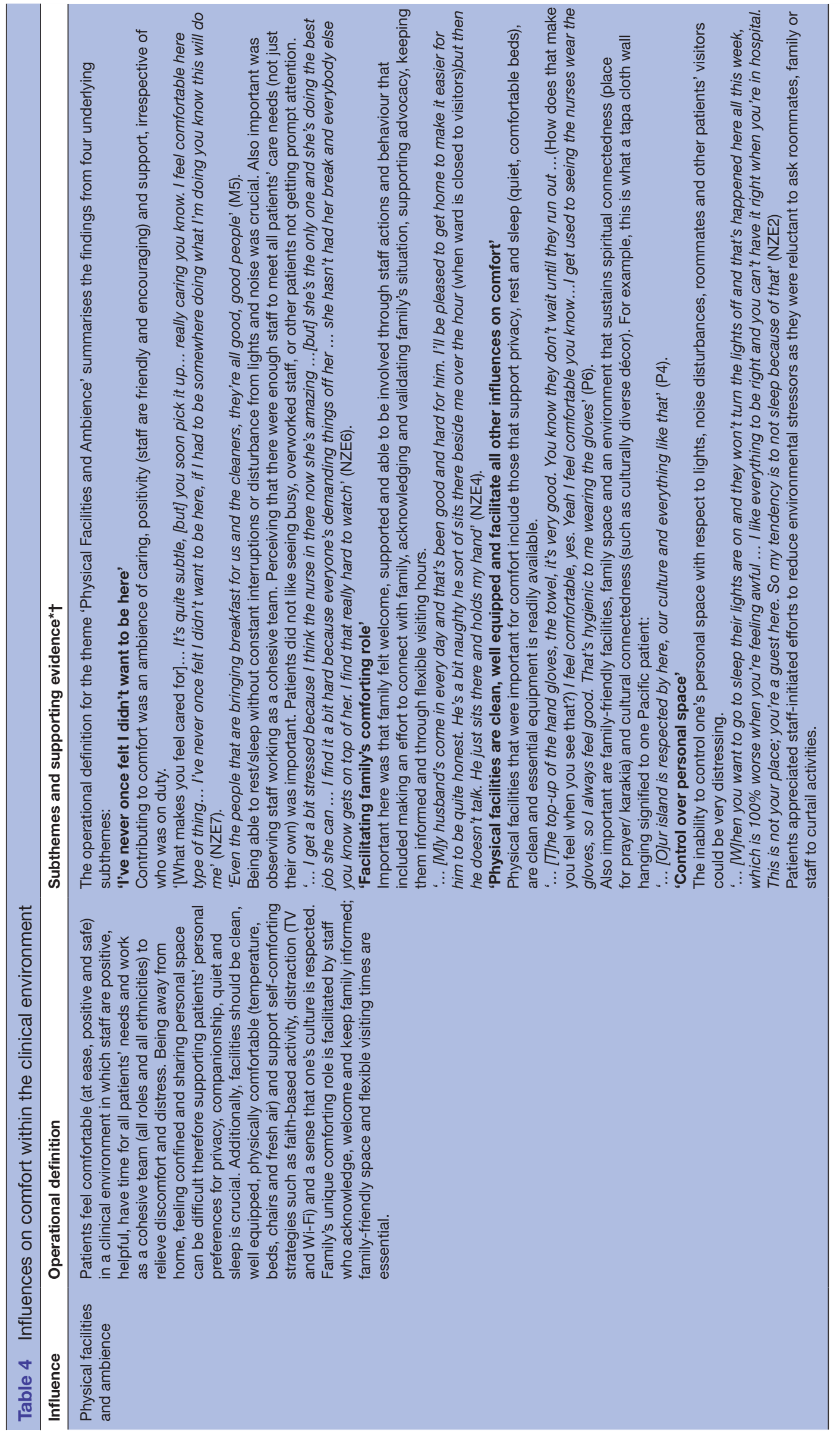

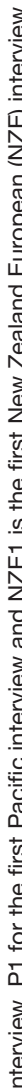




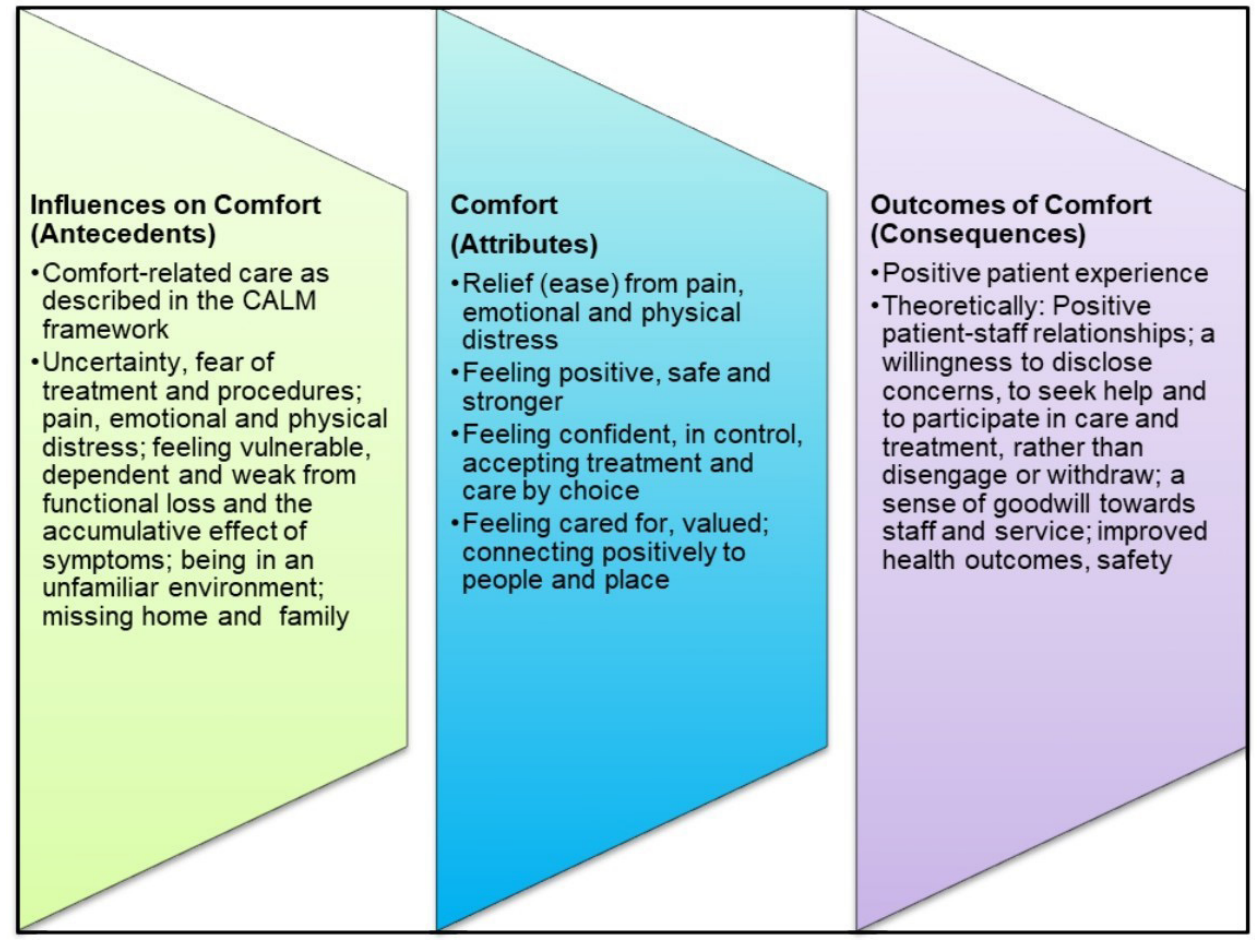

Figure 2 Influences, attributes and outcomes of comfort. CALM, Comfort ALways Matters.

important comfort-related care. Operational definitions for each theme reflect the essence of care that matters to patients and the integrated nature of this care.

Our definition of comfort broadly aligns with others $^{81011}$ in the sense that comfort is defined as a dynamic and multidimensional state. Similarly, nurse theorists, ${ }^{833-37}$ multiple qualitative studies ${ }^{24}$ and concept analyses $^{9} 1012 \quad 23 \quad 38-40$ have consistently described the holistic dimensions of comfort and the art of comforting that we believe are captured in our findings. However, the CALM framework differs from most comfort frameworks/models ${ }^{21} 41-48$ in that patients' perspectives of all influencing factors are captured in one unifying framework. Differentiating the definition of comfort (the state) from the process of comforting (influencing factors) meant that findings are presented as a more 'tangible product' considered essential for implementing qualitative findings into practice ${ }^{49}$ (p. 765). Operational definitions are generated from rich, in-depth data using methods explicitly exploring patients' perspectives. We suggest these definitions provide a clearer direction for practice and quality improvement than other published frameworks. ${ }^{21} 41-4850$

\section{Implications for practice and quality improvement}

Improving patients' experiences of care is core to healthcare quality. Patient experience is defined as 'the sum of all interactions, shaped by an organization's culture, that influence patient perceptions across the continuum of care $^{51}$ (p. 10). Improving patient experience, therefore, requires an understanding of what matters to patients during their interactions with healthcare staff. Work in this area has resulted in a range of frameworks and guiding principles. ${ }^{6} 5253$ Comfort-related care incorporates many factors considered important for patient experience ${ }^{54}$ including compassionate care ${ }^{55} 56$; compassion most simply described as 'the recognition of and response to the distress and suffering of others ${ }^{56}$ (p. 310).

One could assume that initiatives aimed at improving patient experience will also improve comfort. However, all patients interviewed had experienced distressing events even though patient experience indicators at the research site suggested a high-level of person-centred care. Similarly, examples of missed nursing care described in the literature (also known as errors of omission or care rationing) $)^{192257-60}$ relate to care patients described as important for comfort, such as position changes, patient surveillance, comforting/talking with patients, pain management, patient teaching and feeling prepared for discharge. These similarities highlight the inextricable link between care promoting comfort and that inherent in high-quality, safe care.

However, improvement targeting causes of missed nursing care is not the only consideration when aiming to maximise patients' comfort. First, important care is not specific to the actions of any discipline or indeed clinical staff. Second, staff (any role) may not be able to provide the care they wish to provide because of factors beyond their control (eg, lack of equipment, unsupportive ward culture and absence of evidence-based symptom protocols). Therefore, the breadth and depth of all that matters indicate that maximising patients' comfort requires an informed and systematic approach aimed at supporting staff to provide the person-centred care they most likely wish to provide. We therefore ask that healthcare leaders consider how the 
CALM framework may be used to drive a culture of care that maximises patient comfort, beginning with the message that comfort-related care is essential work ${ }^{197-61}$ encompassing a caring, compassionate response to human distress ${ }^{54-56}$ for which healthcare leaders have accountability to promote, monitor and address omissions.

Three principles underpin application of the CALM framework. The first is appreciating the context-specific nature of comfort, meaning that the detail of care underlying each of the broad influences may differ by condition, ethnicity and age. For example, effective symptom management is crucial for comfort, but distressing symptoms may fluctuate by type and stage of a condition. Similarly, family influenced the comfort of patients of all ethnicities but how patients define family and comforting activities differs by ethnicity, age and stage of condition. ${ }^{31}$ The second is that individualised care underpins all operational definitions. Efforts to reduce unwarranted variability through standardised care must not be at the expense of the intuitive art of comforting. The third is that all staff can comfort (or distress). Therefore, consider actions of clinical and ancillary staff when applying the framework. Operational definitions can be used to guide conversations with patients, family and staff about their perception of important care for each influence, with identified gaps providing a basis for improvement work.

\section{Transferability}

Triggers for the need for comforting care identfed in stage 2 were consistent with those identified in other inpatient settings. ${ }^{24}{ }^{31}$ Similarly, the definition of comfort and the CALM framework appear applicable to a range of inpatient populations. Transferability is suggested on the basis that patients of different clinical conditions, age, ethnicity and from a range of inpatient settings within 15 countries $^{24}$ held similar perspectives on the meaning of comfort and the care that influenced it.

\section{Strengths and limitations}

A comprehensive conceptual framework ${ }^{24}$ focused the exploration of patients' perspectives in a clinical setting. Definitions are data derived and represent patients' perspectives. Our method enabled categorisation of concept characteristics in a way that promotes translation into practice; upwards of 60 attributes of comfort and comforting have been previously identified. ${ }^{10}$ This is the first study that has set out to explore a cultural dimension of comfort. Findings collectively represent perspectives held by Māori, Pacific and NZE participants, suggesting that the CALM framework encompasses culturally responsive care. Importantly, within the CALM framework, the patient determines the extent to which culturally safe care is being provided through their sense of feeling 'Culturally connected', that is, they and their family feel welcome; actions and behaviours of others indicate understanding and respect for one's cultural norms and values. This emphasis is consistent with the notion of unsafe cultural practice as 'any action which diminishes, demeans or disempowers the cultural identity and well being of an individual'. ${ }^{62}$

Recruitment stopped when we reached an understanding of how perspectives on comfort broadly differ by ethnicity. However, more can be learnt of the underlying detail for each influencing factor, such as preferences for comforting staff behaviour, attitudes to pain management or body modesty. In accordance with Morse's view, ${ }^{63}$ data saturation on all possible context-specific or individual details was not our intent. Peer debriefing by experienced qualitative researchers throughout all stages of the analysis, Māori and Pacific consultation, prolonged engagement (1082 min of interview), negative case analysis and triangulation methods ${ }^{27}$ promote credibility of the findings. Triangulation-using multiple data sources to produce understanding - was used in both stages of this research. Stage 1 compared findings generated from theoretical and qualitative research (methods triangulation) and involving people from a range of healthcare settings, ages and ethnicities spanning decades of healthcare (triangulation of sources). ${ }^{27}$ Further triangulation occurred in stage 2 when patient interview data were contrasted with findings from the integrative review and included studies. ${ }^{31}$ Concept clarification was sought during all interviews. ${ }^{27}$ However, a limitation is that participants were not asked to comment on the findings.

\section{Implications for research}

Replication of this research may lead to further refinements of operational definitions, evaluate claims of transferability and build an evidence base of context-specific care. Exploring staff perspectives on comfort and determinants of comfort-related care in healthcare settings will inform implementation strategies. Research is also required to identify how the art of comforting can be taught and modelled in clinical practice and educational curricula.

The influence of comfort on patients' outcomes may go beyond patients' experiences of care (see figure 2). Our interview data indicate that a sense of comfort during one's healthcare interaction is associated with positive patient-staff relationships, a willingness to disclose concerns, to seek help and to participate in care and treatment, rather than disengage or withdraw. Other qualitative studies exploring comfort have proposed similar outcomes. ${ }^{24} 64$ An informed, systematic approach to maximising patients' comfort may, therefore, improve not only patients' experiences but also population health, particularly in vulnerable sections of the population. These potential benefits warrant further evaluation. Clinically relevant metrics for quantifying comfort and monitoring important aspects of care are also needed.

\section{CONCLUSION}

This research provides new insights into what comfort means to patients, the care required to promote their comfort and the reasons for which doing so is important. We have developed a definition of comfort and the CALM 
framework, which can be used by healthcare leaders and clinicians to guide practice and quality initiatives aimed at maximising comfort and minimising distress in a range of inpatient populations. A focus on comfort by individuals is crucial, but leadership will be essential for driving the changes needed to reduce unwarranted variability in care that affects comfort.

\section{Author affiliations}

${ }^{1}$ School of Nursing, Faculty of Medical and Health Sciences, The University of Auckland, Auckland, New Zealand

${ }^{2}$ Centre for Quality and Patient Safety Research - Epworth HealthCare Partnership, School of Nursing and Midwifery, Deakin University, Burwood, Victoria, Australia ${ }^{3}$ Faculty of Medical \& Health Sciences, The University of Auckland, Auckland, New Zealand

${ }^{4}$ Auckland City Hospital, Auckland, New Zealand

\section{Twitter Cynthia Wensley @brown_acre}

Acknowledgements The authors would like to thank all staff in the Cardiac Surgical Unit, Auckland City Hospital, He Kamaka Waiora, Māori Health and Pacific Health, Auckland City Hospital, Auckland New Zealand, for supporting this research. The authors would also like to thank the patients who generously agreed to participate in interviews and share their experiences of care, without whom this research would not have been possible.

Contributors CW contributed to conceptualisation of the project, research design, undertook data collection, analysis, wrote the first draft of the manuscript and coordinated its multiple revisions. MB contributed to conceptualisation of the project, research design, analysis and interpretation of the data and critical revision of the manuscript. AM contributed to research design, analysis and interpretation of the data and critical revision of the manuscript. AFM contributed to conceptualisation of the project, research design, interpretation of stage 1 data and critical revision of the manuscript.

Funding This work was supported by an Australian Postgraduate Award (to CW) and a Deakin University Postgraduate Research Scholarship, Australia (to CW).

\section{Competing interests None declared.}

Patient and public involvement Patients and/or the public were involved in the design, or conduct, or reporting, or dissemination plans of this research. Refer to the Methods section for further details.

Patient consent for publication Not required.

Ethics approval Ethics approval was gained from Deakin University Human Research Ethics Committee (2013-180), the New Zealand Health and Disability Ethics Committee (13/CEN/95) and the institution at which recruitment and interviewing occurred $(A+5824)$.

Provenance and peer review Not commissioned; externally peer reviewed.

Data availability statement Consistent with our institution's ethics approval, no additional data generated in this study can be made available.

Open access This is an open access article distributed in accordance with the Creative Commons Attribution Non Commercial (CC BY-NC 4.0) license, which permits others to distribute, remix, adapt, build upon this work non-commercially, and license their derivative works on different terms, provided the original work is properly cited, appropriate credit is given, any changes made indicated, and the use is non-commercial. See: http://creativecommons.org/licenses/by-nc/4.0/.

\section{ORCID iD}

Cynthia Wensley http://orcid.org/0000-0002-6224-7514

\section{REFERENCES}

1 Picker Institute Europe. About Us: Our history \& impact. Available: https://www.picker.org/about-us/our-history-impact/ [Accessed Nov 2019].

2 Charmel PA, Frampton SB. Building the business case for patientcentered care. Healthc Financ Manage 2008;62:80-5.

3 Committee on Quality of Health Care in America, Institute of MedicineKohn LT, Corrigan J, Donaldson MS, eds. To err is human: building a safer health system. Washington, D.C: National Academy Press, 2000.

4 National Institute for Health and Clinical Excellence. Patient experience in adult NHS services: improving the experience of care for people using adult NHS services clinical guidance, 2012. Available: https://www.nice.org.uk/guidance/cg138/evidence/fullguideline-pdf-185142637 [Accessed Nov 2019].

5 Gerteis M, Edgman-Levitan S, Daley J. Through the patient's eyes: understanding and promoting patient-centered care, 1993.

6 Picker Institute Europe. Principles of patient centred care 2017, 2017. Available: http://www.picker.org/about-us/principles-ofpatient-centred-care/ [Accessed May 2017].

7 Institute of Medicine, Committee on Quality of Health Care in America. Crossing the quality chasm: a new health system for the 21st century. Washington, DC: National Academy Press, 2001.

8 Kolcaba K. Comfort theory and practice. New York: Springer Publishing company, 2003.

9 Lowe LM, Cutcliffe JR. A concept analysis of comfort. In: Cutliffe JR, McKenna HP, eds. The essential concepts of nursing: building blocks for practice. London: Elsevier, 2005.

10 Siefert ML. Concept analysis of comfort. Nurs Forum 2002;37:16-23.

11 Morse JM. Comfort: the refocusing of nursing care. Clin Nurs Res 1992;1:91-106.

12 Tutton E, Seers K. An exploration of the concept of comfort. J Clin Nurs 2003;12:689-96.

13 Lin Y-P, Watson R, Tsai Y-F. Dignity in care in the clinical setting: a narrative review. Nurs Ethics 2013;20:168-77.

14 Baillie L. Patient dignity in an acute hospital setting: a case study. Int J Nurs Stud 2009;46:23-37.

15 Gray A, Cox J. The roots of compassion and empathy: implementing the Francis report and the search for new models of health care. Eur $J$ Pers Cent Healthc 2015;3:122-30.

16 The Mid Staffordshire NHS Foundation Trust Inquiry. Independent inquiry into care provided by mid Staffordshire NHS Foundation trust; 2010. https://www.gov.uk/government/publications/independentinquiry-into-care-provided-by-mid-staffordshire-nhs-foundationtrust-january-2001-to-march-2009

17 Bauer A. First, do no harm: the patient's experience of avoidable suffering as harm. Patient Exp J 2018;5:13-15.

18 Dixon-Woods M, Baker R, Charles K, et al. Culture and behaviour in the English National health service: overview of lessons from a large multimethod study. BMJ Qual Saf 2014;23:106-15.

19 Lake ET, Germack HD, Viscardi MK. Missed nursing care is linked to patient satisfaction: a cross-sectional study of US hospitals. BMJ Qual Saf 2016;25:535-43.

20 Allen T, Rieck T, Salsbury S. Patient perceptions of an AIDET and hourly rounding program in a community hospital: results of a qualitative study. Patient Exp J 2016;3:42-9.

21 Tutton E, Seers K. Comfort on a ward for older people. J Adv Nurs 2004; 46:380-9.

22 Aiken LH, Sloane DM, Ball J, et al. Patient satisfaction with hospital care and nurses in England: an observational study. BMJ Open 2018;8:e019189.

23 Pinto S, Caldeira S, Martins JC, et al. Evolutionary analysis of the concept of comfort. Holist Nurs Pract 2017;31:243-52.

24 Wensley C, Botti M, McKillop A, et al. A framework of comfort for practice: an integrative review identifying the multiple influences on patients' experience of comfort in healthcare settings. Int J Qual Health Care 2017;29:151-62.

25 Sandelowski M. What's in a name? qualitative description revisited. Res Nurs Health 2010;33:77-84.

26 Ball K, Swallow V. Patient experiences of cardiac surgery and nursing care: a narrative review. British Journal of Cardiac Nursing 2016;11:348-58.

27 Lincoln YS, Guba EG. Naturalistic inquiry. Beverly Hills: Calif: Sage Publications, 1985: 9. 438-9.

28 Thomas DR. A general inductive approach for analyzing qualitative evaluation data. Am J Eval 2006;27:237-46.

29 Braun V, Clarke V. Using thematic analysis in psychology. Qual Res Psychol 2006;3:77-101.

30 Ritchie J, Lewis J, McNaughton Nicholls C, et al. Qualitative research practice: a guide for social science studers and researchers. Los Angeles, California: Sage, 2014.

31 Wensley C. A multidimensional framework of comfort for practice and quality improvement, 2018: 312. http://dro.deakin.edu.au/view/DU: 30111035

32 O'Brien BC, Harris IB, Beckman TJ, et al. Standards for reporting qualitative research: a synthesis of recommendations. Acad Med 2014;89:1245-51. 
33 Orlando IJ. The dynamic nurse-patient relationship: function, process, and principles. New York: National League for Nursing, 1990.

34 Paterson J, Zderad L. Humanistic nursing, 1976. http://www. gutenberg.org/files/25020/25020-8.txt

35 Hall LE. The Loeb center for nursing and rehabilitation, Montefiore hospital and medical center, Bronx, New York. Int J Nurs Stud 1969;6:81-97.

36 Watson J. Nursing the philosophy and science of caring, revised edition. Boulder University Press of Colorado, 2008. http://www.jstor. org/stable/j.ctt1d8h9wn

37 Leininger MM. Culture care diversity and universality: a theory of nursing. New York: National League for Nursing Press, 1991.

38 Kolcaba KY, Kolcaba RJ. An analysis of the concept of comfort. J Adv Nurs 1991;16:1301-10.

39 Malinowski A, Stamler LL. Comfort: exploration of the concept in nursing. J Adv Nurs 2002;39:599-606.

40 Oliveira I. Comfort measures: a concept analysis. Res Theory Nurs Pract 2013;27:95-114.

41 Williams AM, Irurita VF. Therapeutic and non-therapeutic interpersonal interactions: the patient's perspective. J Clin Nurs 2004;13:806-15.

42 Williams AM, Irurita VF. Enhancing the therapeutic potential of hospital environments by increasing the personal control and emotional comfort of hospitalized patients. Appl Nurs Res 2005;18:22-8.

43 Williams AM, Irurita VF. Emotional comfort: the patient's perspective of a therapeutic context. Int J Nurs Stud 2006;43:405-15.

44 Williams AM, Dawson S, Kristjanson LJ. Exploring the relationship between personal control and the hospital environment. J Clin Nurs 2008;17:1601-9.

45 Chau L, Hegedus L, Praamsma M, et al. Women living with a spinal cord injury: perceptions about their changed bodies. Qual Health Res 2008;18:209-21.

46 Yousefi H, Abedi HA, Yarmohammadian $\mathrm{MH}$, et al. Comfort as a basic need in hospitalized patients in Iran: a hermeneutic phenomenology study. J Adv Nurs 2009;65:1891-8.

47 Coelho A, Parola V, Escobar-Bravo M, et al. Comfort experience in palliative care: a phenomenological study. BMC Palliat Care 2016;15:1-8.

48 Morse JM, Havens GA, Wilson S. The comforting interaction: developing a model of nurse-patient relationship. Sch Inq Nurs Pract 1997;11:321-47.

49 Morse JM. Towards understanding comfort and comforting. In: Analyzing and Conceptualizing the theoretical foundations of nursing. New York: United States Springer Publishing Company, 2017: 675-98.

50 Kolcaba KY. Holistic comfort: operationalizing the construct as a nurse-sensitive outcome. ANS Adv Nurs Sci 1992;15:1-10.

51 Wolf JA, Niederhauser V, Marshburn D, et al. Defining patient experience. Patient Exp J 2014;1:7-19.

52 Institute for Healthcare Improvement. Person- and Family-Centered care 2015. Available: http://www.ihi.org/Topics/PFCC/Pages/ Overview.aspx [Accessed Jul 2018].

53 The Beryl Institute. Experience framework. Available: https://www. theberylinstitute.org/page/ExperienceFramework [Accessed Jul 2018].

54 Wolf JA. To care is human: the factors influencing human experience in healthcare today 2018. Available: https://www.theberylinstitute. org/page/ToCarelsHuman [Accessed Jan 2019].

55 Durkin J, Usher K, Jackson D. Embodying compassion: a systematic review of the views of nurses and patients. J Clin Nurs 2019;28:1380-92.

56 Lown BA, Mclntosh S, Gaines ME, et al. Integrating Compassionate, Collaborative Care (the "Triple C") Into Health Professional Education to Advance the Triple Aim of Health Care. Acad Med 2016;91:310-6.

57 Ball JE, Murrells T, Rafferty AM, et al. 'Care left undone' during nursing shifts: associations with workload and perceived quality of care. BMJ Qual Saf 2014;23:116-25.

58 Ball JE, Bruyneel L, Aiken LH, et al. Post-Operative mortality, missed care and nurse staffing in nine countries: a cross-sectional study. Int J Nurs Stud 2018;78:10-15.

59 Kalisch BJ, Landstrom G, Williams RA. Missed nursing care: errors of omission. Nurs Outlook 2009;57:3-9.

60 New Zealand Nurses Organisation. NZNO position statement on care rationing 2014. Available: http://www.nzno.org.nz/carerationing [Accessed Jul 2017]

61 Feo R, Conroy T, Jangland E, et al. Towards a standardised definition for fundamental care: a modified Delphi study. J Clin Nurs 2018;27:2285-99.

62 Nursing Council of New Zealand. Guidelines for cultural safety, the Treaty of Waitangi and Māori health in nursing education and Practice2011. Available: http://nursingcouncil.org.nz/Publications/ Standards-and-guidelines-for-nurses [Accessed Jul 2018].

63 Morse JM. "Data were saturated ...". Qual Health Res 2015;25:587-8

64 Zelazny SM, Chang JC, Burke JG, et al. Adolescent and young adult women's recommendations for establishing comfort with family planning providers' communication about and assessment for intimate partner violence. J Commun Healthc 2019;12:32-43. 